\title{
B physics and Quarkonia in CMS
}

\author{
Sara Fiorendi ${ }^{1, a}$ on behalf of the CMS Collaboration \\ ${ }^{1}$ Università degli Studi di Milano - Bicocca and INFN
}

\begin{abstract}
The heavy-flavor sector offers the opportunity to perform indirect tests of beyond the Standard Model physics through precision measurements and of quantum chromodynamics $(\mathrm{QCD})$ through particle production studies. A review of recent measurements on heavy flavors from the CMS experiment is presented. Results are based on data collected during LHC Run I and Run II and include measurements of heavy flavor production and properties, rare decays, $\mathrm{CP}$ violation, exotic and standard quarkonia.
\end{abstract}

\section{Introduction}

The heavy flavor program of the CMS experiment [1] at the CERN LHC is very competitive and differentiated. The main objectives include testing the Standard Model (SM) predictions via high precision measurements of heavy flavor properties (decay rates, lifetimes, CP violation) and production spectra, searching for new exotic quarkonia states, new hadrons and indications of new physics through rare decay studies. Selected recent results, based on data collected at 8 and $13 \mathrm{TeV}$, are reviewed in this Letter.

\section{Measurement of $\mathrm{B}^{+}$production cross section at $13 \mathrm{TeV}$}

The CMS measurement of the $\mathrm{B}^{+}$production cross section at $13 \mathrm{TeV}[6]$ provides a test of theoretical calculations, complementing the measurements carried out at 7 and $8 \mathrm{TeV}$ by the CMS [3], LHCb [4, 5] and ATLAS [2] Collaborations.

The inclusive $\mathrm{B}^{+}$differential production cross sections as a function of the $\mathrm{B}^{+}$meson transverse momentum and rapidity are measured using $50.8 \mathrm{pb}^{-1}$ of data collected in 2015 with $50 \mathrm{~ns}$ bunch spacing. The measurement is based on the inclusive channel $\mathrm{pp} \rightarrow \mathrm{B}^{+} \mathrm{X} \rightarrow \mathrm{J} / \psi \mathrm{K}^{+} \mathrm{X}$, where $\mathrm{J} / \psi \rightarrow \mu^{+} \mu^{-}$, and covers the phase space region where $10<\mathrm{p}_{\mathrm{T}}^{\mathrm{B}}<100 \mathrm{GeV}$ and $\left|\mathrm{y}^{\mathrm{B}}\right|<2.4$. Data have been collected using a displaced dimuon trigger path, which requires two muons coming from the same vertex and with invariant mass compatible with the $\mathrm{J} / \psi$ one. Candidate $\mathrm{B}^{+}$mesons are reconstructed by combining a $\mathrm{J} / \psi$ with an additional track, assumed to be a kaon.

The detection efficiency and geometrical acceptance are evaluated through a Monte Carlo simulation, while trigger and reconstruction efficiencies are measured directly from data.

The signal yield is extracted with an extended unbinned maximum likelihood fit to the invariant mass distribution of the $\mathrm{B}^{+}$candidates, in each $\mathrm{p}_{\mathrm{T}}^{\mathrm{B}}$ or $\left|\mathrm{y}^{\mathrm{B}}\right|$ bins. The signal component is modeled by

a e-mail: sara.fiorendi@ cern.ch 
the sum of two Gaussians functions, while the combinatorial background component is described by an exponential function. The background from mis-reconstructed $\mathrm{B} \rightarrow \mathrm{J} / \psi \mathrm{K}^{+} \mathrm{X}$ decays is modeled by an error function.

Systematic uncertainties have been evaluated on signal yield extraction, $\mathrm{p}_{\mathrm{T}}$ and $\mathrm{y}$ resolution, $\mathrm{p}_{\mathrm{T}}^{\mathrm{B}}$ and $\left|\mathrm{y}^{\mathrm{B}}\right|$ simulated distributions, $\mathrm{B}^{+}$lifetime, trigger and muon/track reconstruction, size of the simulation, and luminosity measurement.

Results for the differential cross sections as a function of $\mathrm{p}_{\mathrm{T}}^{\mathrm{B}}$, integrated within $\left|\mathrm{y}^{\mathrm{B}}\right|<2.4$, and as a function of $\left|\mathrm{y}^{\mathrm{B}}\right|$, integrated within $10<\mathrm{p}_{\mathrm{T}}^{\mathrm{B}}<100 \mathrm{GeV}$, are shown in Fig. 1, together with the corresponding $7 \mathrm{TeV}$ measurements [3]. FONLL (shaded boxes) and PYTHIA (dashed lines) calculations are also reported on the plots, showing reasonable agreement both in terms of shape and normalization.
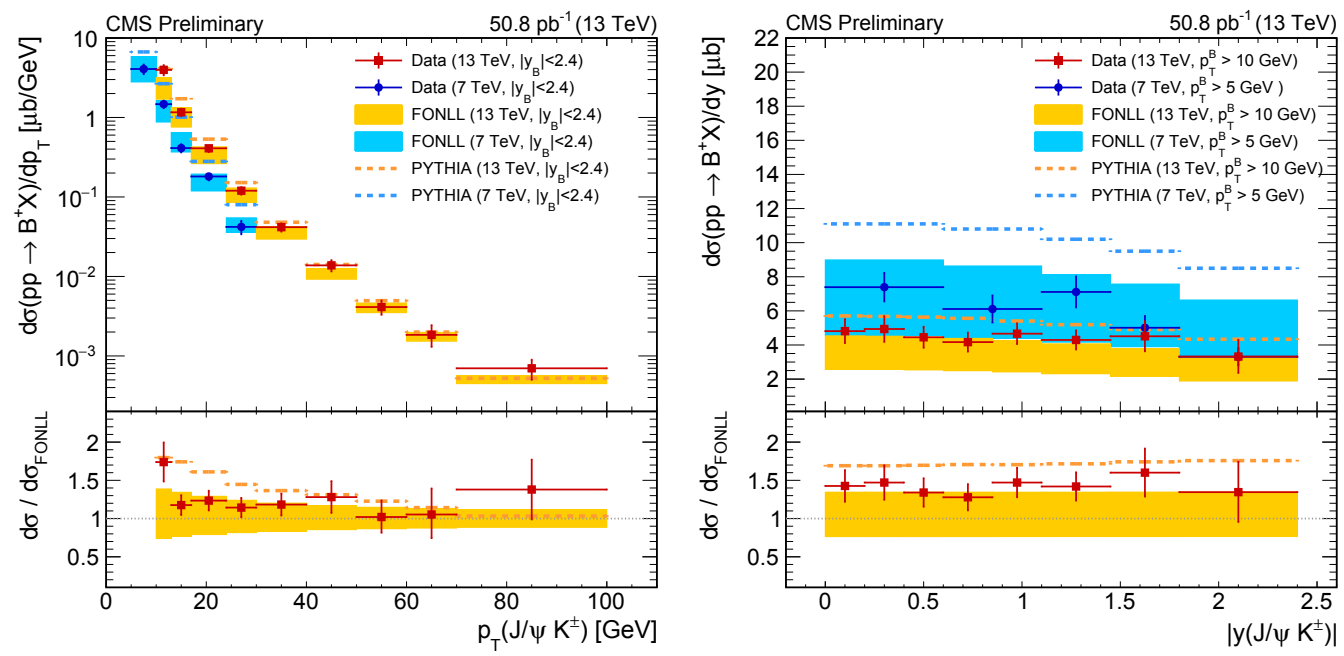

Figure 1. $\mathrm{B}^{+}$differential production cross sections at $13 \mathrm{TeV}$ as a function of $\mathrm{p}_{\mathrm{T}}^{\mathrm{B}}$ for $\left|\mathrm{y}^{\mathrm{B}}\right|<2.4$, and rapidity for $10<\mathrm{p}_{\mathrm{T}}^{\mathrm{B}}<100 \mathrm{GeV}$. The previous CMS measurements from $7 \mathrm{TeV}$ data [3] (circles) are also shown. The vertical bars show the total uncertainty in the measured cross sections, and the horizontal bars represent the bin width. The calculations from FONLL and PYTHIA are shown as shaded boxes and dashed lines, respectively. The bottom panels display the ratio of the data at $13 \mathrm{TeV}$ to the FONLL predictions (points) and the ratios of the PYTHIA to the FONLL calculations (dashed lines), with the shaded region displaying the uncertainties in the FONLL predictions.

\section{Quarkonium production cross section at $13 \mathrm{TeV}$}

Precise measurements of cross sections and polarizations for five quarkonium states $(\mathrm{J} / \psi, \psi(2 \mathrm{~S}), \mathrm{Y}(\mathrm{nS}))$ were provided by the LHC experiments already during Run I [7-16]. Measurements at $13 \mathrm{TeV}$ offer an interesting opportunity to test Non-Relativistic QCD at higher energies and extend the comparison to theoretical calculations to higher $\mathrm{p}_{\mathrm{T}}$ ranges.

The analysis [17] is based on data collected in 2015, corresponding to an integrated luminosity of $2.4 \mathrm{fb}^{-1}$ for $\mathrm{J} / \psi$, and $2.7 \mathrm{fb}^{-1}$ for the other mesons. Quarkonium states are reconstructed through their decay into two opposite-sign muons. The measurement is restricted to the central region, where $\left|\eta^{\mu \mu}\right|<1.25$. 
The acceptance is evaluated through simulated samples, assuming unpolarized dimuons. Muon reconstruction, identification and trigger efficiencies and dimuon efficiency are measured on data as a function of muon (dimuon) $\mathrm{p}_{\mathrm{T}}$ and pseudorapidity.

Yields are extracted through an unbinned maximum likelihood fit to the dimuon invariant mass distribution. The three $\mathrm{Y}(\mathrm{nS})$ signal peaks are modeled by Crystal Ball functions, and the background is described by an exponential distribution. For Charmonium states, the additional non-prompt component, originating from b-hadron decays, is taken into account. A simultaneous fit to mass and pseudo proper-decay-length distributions is used to disentangle the amount of prompt and non-prompt mesons.

Systematic uncertainties on dimuon acceptance and efficiencies, determination of the signal yields and luminosity measurement are evaluated.

Cross section results for the $\mathrm{J} / \psi$ and $\psi(2 \mathrm{~S})$ states are shown in the left plot of Fig. 2 as a function of the dimuon $\mathrm{p}_{\mathrm{T}}$, together with the corresponding $7 \mathrm{TeV}$ measurements, for the rapidity-integrated range $|\mathrm{y}|<1.2$. The right plot shows the cross section measurements for the $\mathrm{Y}(\mathrm{nS})$ states. The $13 \mathrm{TeV}$ cross sections of all five quarkonium states are factors of 2 to 3 larger than the corresponding $7 \mathrm{TeV}$ cross sections, with slow changes as a function of the dimuon $\mathrm{p}_{\mathrm{T}}$.
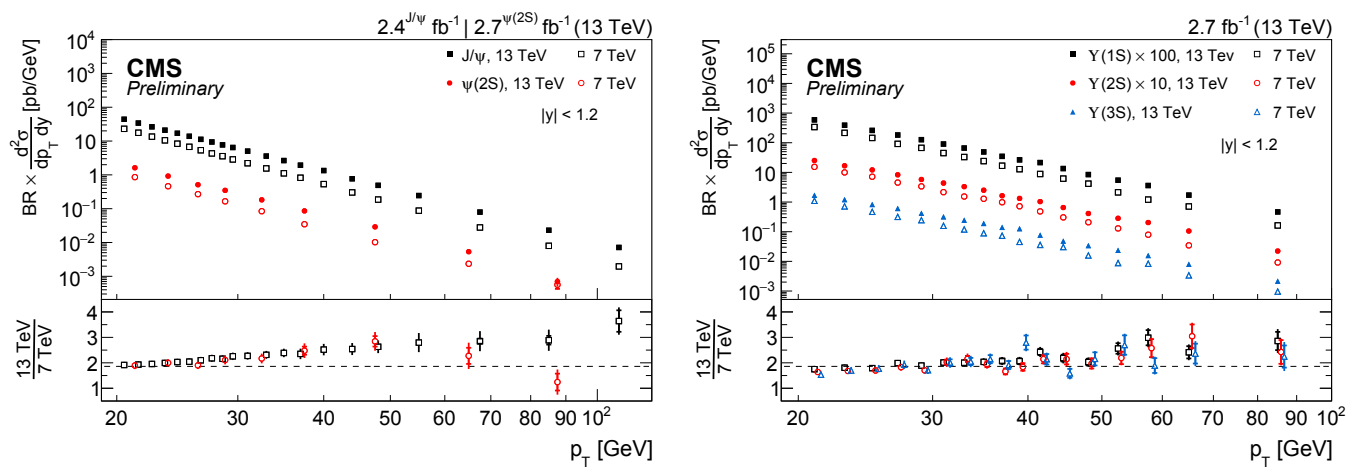

Figure 2. Differential cross sections times branching ratios for $7 \mathrm{TeV}$ and $13 \mathrm{TeV} \mathrm{CMS}$ data [17], for $\mathrm{J} / \psi$ and $\psi(2 S)$ (left) and $\mathrm{Y}(\mathrm{nS})$ (right), for $|\mathrm{y}|<1.2$. The inner error bars represent the statistical uncertainty while the total errors show the statistical and systematic uncertainties. The uncertainty on the luminosity measurement is not included in the plots.

\section{Observation of $Y(1 S)$ pair production}

The first observation of the simultaneous production of $\mathrm{Y}(1 \mathrm{~S})$ pairs has been reported by CMS in [18]. Measurements of the production of quarkonium pairs are considered the first step in the search for tetra-quark states. Though the event yield of this observation is limited, future measurements with higher statistics can provide information on the production mechanism (Single or Double Parton Scattering).

The analysis is based on $20.7 \mathrm{fb}^{-1}$ of data collected in 2012 at $\sqrt{s}=8 \mathrm{TeV}$. Events are selected by requiring four muons with zero total charge, each muon having $\mathrm{p}_{\mathrm{T}}>3.5 \mathrm{GeV}$. Each Y candidate is reconstructed by performing a kinematic fit to the oppositely charged muon pairs and requiring a minimum vertex fit $\chi^{2}$ probability. Two kinematic variables are defined: the dimuon invariant mass of the higher mass $\mathrm{Y}$ candidate, $\mathbf{M}_{\mu \mu}^{1}$ and that of the lower mass $\mathrm{Y}$ candidate, $\mathbf{M}_{\mu \mu}^{2}$. 
The signal yields of $\mathrm{Y}(1 \mathrm{~S}) \mathrm{Y}(1 \mathrm{~S})$ and $\mathrm{Y}(1 \mathrm{~S}) \mathrm{Y}(2 \mathrm{~S})$ are extracted through a two-dimensional unbinned maximum likelihood fit to $\mathbf{M}_{\mu \mu}^{1}$ and $\mathbf{M}_{\mu \mu}^{2}$ (Fig. 3). The invariant mass distribution of each muon pair is modeled by a signal and background component, the signal model consisting in the sum of two Crystal Ball functions, and the background in a first order Chebychev Polynomial. The signal yield of $\mathrm{Y}(1 \mathrm{~S}) \mathrm{Y}(1 \mathrm{~S})$ is measured to be $38 \pm 7$ events and the statistical significance for $\mathrm{pp} \rightarrow$ $\mathrm{Y}(1 \mathrm{~S}) \mathrm{Y}(1 \mathrm{~S})$ production is determined to be 9.6 standard deviations.

The inclusive cross section is measured in the region $|\mathrm{y}(\mathrm{Y})|<2.0$ and $\mathrm{p}_{\mathrm{T}}(\mathrm{Y})<50 \mathrm{GeV}$. Efficiency and acceptance corrections are evaluated on an event-by-event basis using data-embedding methods to minimize the possible model dependence. Several sources of systematic uncertainties have been considered: shape of the signal model, uncertainties on trigger and reconstruction efficiency and acceptance, luminosity measurement.

Assuming isotropic $\mathrm{Y}(1 \mathrm{~S})$ decays into muons, the total cross section of $\mathrm{Y}(1 \mathrm{~S})$ pair production is measured to be $\sigma_{T}=68.8 \pm 12.7$ (stat) \pm 7.4 (syst) \pm 2.8 (BR) pb. It is also measured that extreme polarization scenarios could vary the results by $+36 \%$ (100\% longitudinal polarization) or $-38 \%$ (100\% transverse polarization).
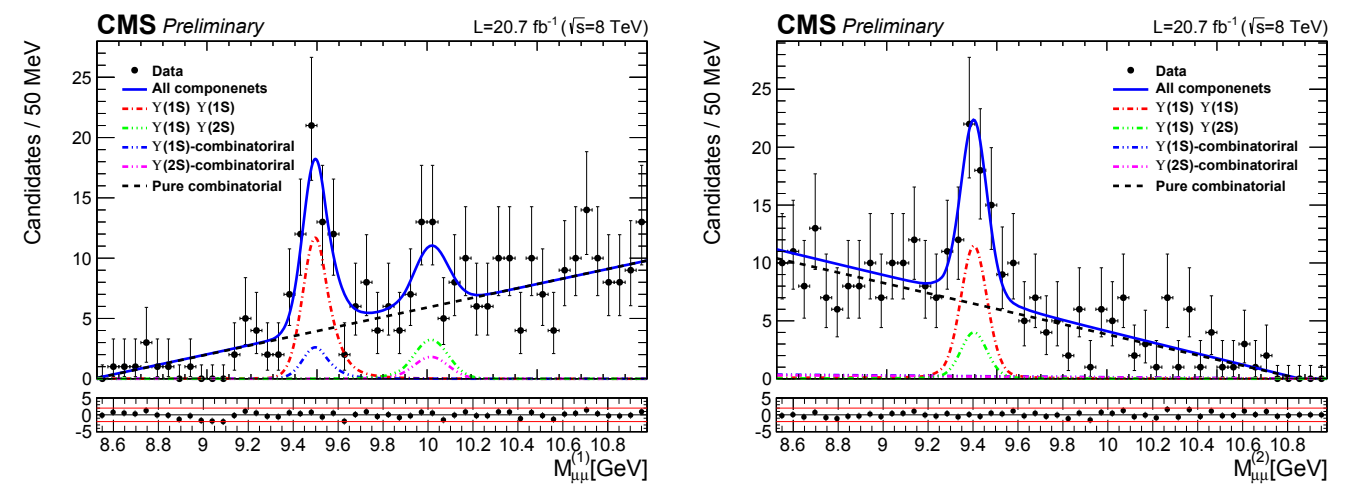

Figure 3. Projections of the two dimensional fit to $\mathrm{M}_{\mu \mu}^{1}$ (left) and $\mathrm{M}_{\mu \mu}^{2}$ (right). The lower pads show the difference between the total fit curve and data, with red lines corresponding to a difference of \pm 2 standard deviations.

\section{Angular analysis of $B^{0} \rightarrow K^{* 0} \mu^{+} \mu^{-}$}

The flavor-changing neutral-current decay $\mathrm{B}^{0} \rightarrow \mathrm{K}^{* 0} \mu^{+} \mu^{-}$, highly suppressed in the SM, is particularly sensitive to new physics contributions. They could reveal themselves through modifications of the decay properties, such as the branching fraction, muon forward-backward asymmetry $\left(\mathrm{A}_{\mathrm{FB}}\right)$ and longitudinal polarization fraction of the $K^{* 0}\left(\mathrm{~F}_{\mathrm{L}}\right)$. These physical parameters are measured by the CMS Collaboration on a $20.5 \mathrm{fb}^{-1}$ data sample collected at $8 \mathrm{TeV}$ [19]. The decays $\mathrm{B}^{0} \rightarrow \mathrm{J} / \psi \mathrm{K}^{* 0}$ and $\mathrm{B}^{0} \rightarrow \psi(2 \mathrm{~S}) \mathrm{K}^{* 0}$ are used as control channels.

The $\mathrm{B}^{0} \rightarrow \mathrm{K}^{* 0} \mu^{+} \mu^{-}$decay is fully described by three angles (the helicity angle for the dimuon system, $\theta_{L}$, the helicity angle of the $\mathrm{K}^{* 0}$ candidate, $\theta_{K}$, and the angle between the $\mathrm{K}^{* 0}$ and dimuon planes, $\phi$ ) and the dimuon invariant mass squared $\left(q^{2}\right)$. The results of the analysis are extracted from unbinned extended maximum likelihood fits, in bins of $q^{2}$, to the invariant mass of the $\mathrm{B}^{0}$ candidate and the two angles $\theta_{K}$ and $\theta_{L}$. In Fig. 4 the results are compared with two sets of SM predictions [20-22], which differ in the calculation of the form factors. Good agreement is observed between data and theoretical calculations. 

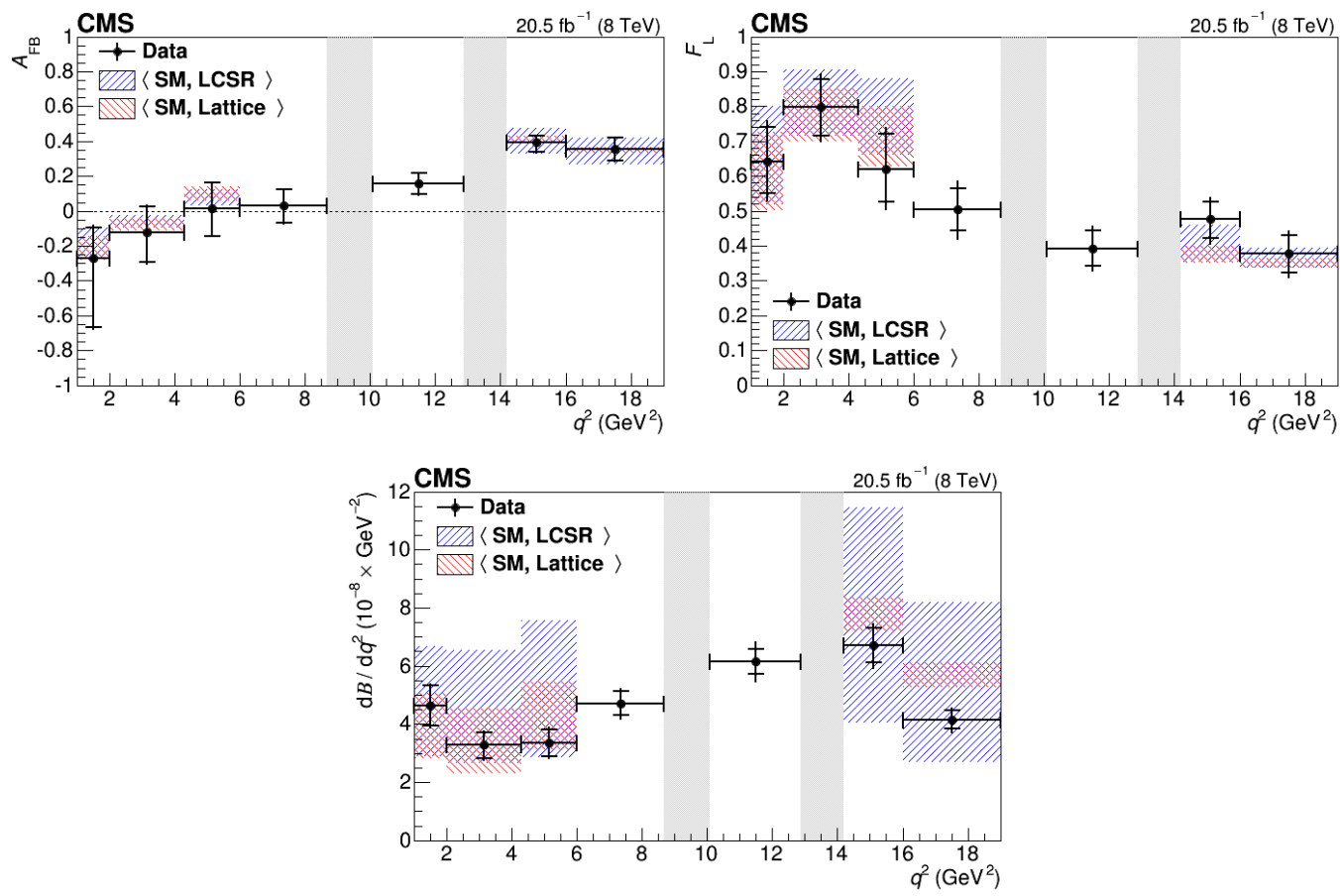

Figure 4. Results of the measurement of $\mathrm{A}_{\mathrm{FB}}, \mathrm{F}_{\mathrm{L}}$ and the differential branching fraction versus the dimuon $q^{2}$. The inner error bars on data points represent the statistical uncertainty, while the total errors show the total uncertainty. The gray shaded regions correspond to the $\mathrm{J} / \psi$ and $\psi(2 S)$ resonances. Two sets of Standard Model predictions [20-22] are also shown.

\section{Measurement of $\phi_{s}$ and $\Delta \Gamma_{s}$}

The interference between direct $\mathrm{B}_{\mathrm{s}}$ decays to $\mathrm{J} / \psi \phi$ and decays through $\mathrm{B}_{\mathrm{s}}^{0}-\overline{\mathrm{B}}_{\mathrm{s}}^{0}$ mixing gives rise to the $\mathrm{CP}$ violating weak phase $\phi_{s}$. The SM predictions for $\phi_{s}$ are very precise, so any measured deviation would be an indication of new physics contributions.

The decay $\mathrm{B}_{\mathrm{s}} \rightarrow \mathrm{J} / \psi \Phi(1020) \rightarrow \mu^{+} \mu^{-} \mathrm{K}^{+} \mathrm{K}^{-}$is exploited to measure $\phi_{s}$ and the decay-width difference $\Delta \Gamma_{s}$ between light and heavy $\mathrm{B}_{\mathrm{s}}$ mass eigenstates [23]. A time-dependent and flavor-tagged angular analysis is used to disentangle the CP-odd and CP-even components of the final state. Three angles are defined to describe the decay: $\theta_{T}\left(\phi_{T}\right)$ is the polar (azimuthal) angle defined by the $\mu^{+}$in the $J / \psi$ rest frame, while the helicity angle $\psi_{T}$ is the angle of the $\mathrm{K}^{+}$in the $\Phi(1020)$ rest frame with respect to the negative $\mathrm{J} / \psi$ momentum direction. The flavor of each $\mathrm{B}_{\mathrm{s}}$ candidate at production time is determined with an opposite-side lepton tagging algorithm.

An unbinned maximum likelihood fit to the data is performed by including the information on the $\mathrm{B}_{\mathrm{s}}$ invariant mass, the three decay angles, the flavor tag decision, the proper decay time ct and its uncertainty.

The analysis is performed on a $20 \mathrm{fb}^{-1}$ dataset collected at $8 \mathrm{TeV}$. The measured values for $\phi_{s}$ and $\Delta \Gamma_{s}$ are $\phi_{s}=-0.075 \pm 0.097$ (stat) \pm 0.031 (syst) rad and $\Delta \Gamma_{s}=0.095 \pm 0.013$ (stat) \pm 0.007 (syst) $\mathrm{ps}^{-1}$. The left plot of Fig. 5 shows the measured values together with SM predictions. The right 
plot shows the combination of the results from different experiments, as provided by the HFAG group [24].
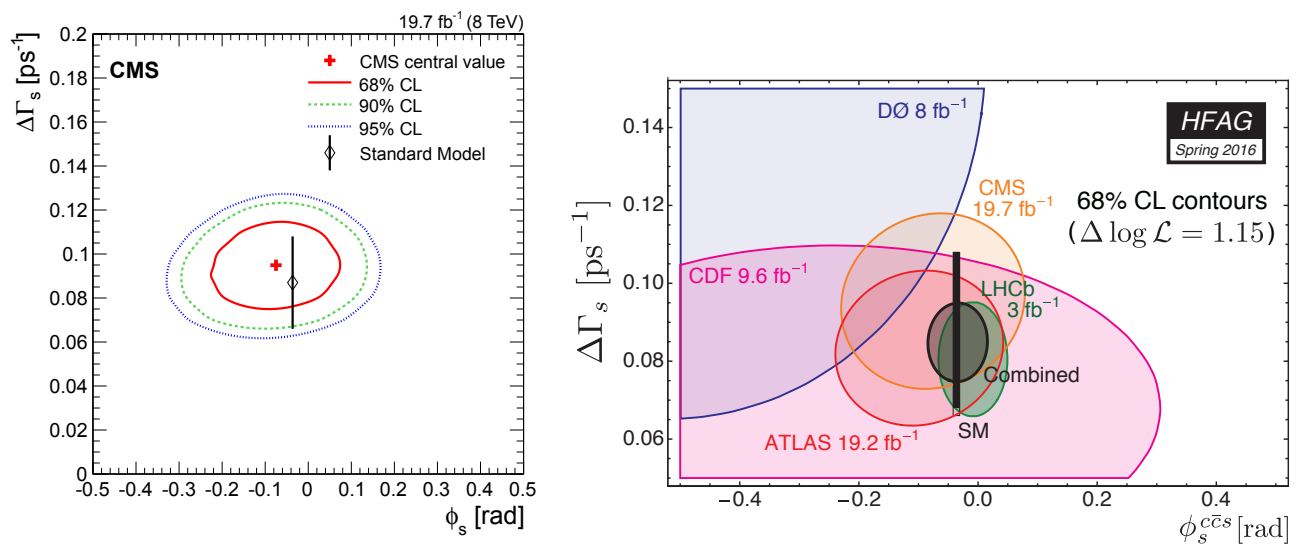

Figure 5. Left: $\phi_{s}$ and $\Delta \Gamma_{s}$ as measured by CMS [23], together with $68 \%, 90 \%$, and $95 \%$ CL contours. SM predictions are also shown. Right: individual 68\% confidence-level contours of ATLAS, CMS, CDF, D0 and LHCb measurements, in the $\left(\phi_{s}^{c c s}, \Delta \Gamma_{s}\right)$ plane [24]. Their combined contour (solid line and shaded area), and the Standard Model predictions (thin black rectangle) are also shown.

\section{Conclusions}

The Run II of the LHC will provide a significant statistics of heavy flavor events, improving the sensitivity reach of many analyses. The first results from CMS on $13 \mathrm{TeV}$ data have been summarized in this Letter, together with selected measurements carried out on Run I data.

\section{References}

[1] S. Chatrchyan et al. [CMS Collaboration], JINST 3 (2008) S08004. doi:10.1088/17480221/3/08/S08004

[2] G. Aad et al. [ATLAS Collaboration], JHEP 1310, 042 (2013) doi:10.1007/JHEP10(2013)042 [arXiv:1307.0126 [hep-ex]].

[3] V. Khachatryan et al. [CMS Collaboration], Phys. Rev. Lett. 106, 112001 (2011) doi:10.1103/PhysRevLett.106.112001 [arXiv:1101.0131 [hep-ex]].

[4] R. Aaij et al. [LHCb Collaboration], JHEP 1204, 093 (2012) doi:10.1007/JHEP04(2012)093 [arXiv:1202.4812 [hep-ex]].

[5] R. Aaij et al. [LHCb Collaboration], JHEP 1308, 117 (2013) doi:10.1007/JHEP08(2013)117 [arXiv:1306.3663 [hep-ex]].

[6] CMS Collaboration, CMS-PAS-BPH-15-004, https://cds.cern.ch/record/2114806

[7] V. Khachatryan et al. [CMS Collaboration], Phys. Rev. D 83, 112004 (2011) doi:10.1103/PhysRevD.83.112004 [arXiv:1012.5545 [hep-ex]].

[8] S. Chatrchyan et al. [CMS Collaboration], Phys. Lett. B 727, 101 (2013) doi:10.1016/j.physletb.2013.10.033 [arXiv:1303.5900 [hep-ex]]. 
[9] V. Khachatryan et al. [CMS Collaboration], Phys. Rev. Lett. 114, no. 19, 191802 (2015) doi:10.1103/PhysRevLett.114.191802 [arXiv:1502.04155 [hep-ex]].

[10] V. Khachatryan et al. [CMS Collaboration], Phys. Lett. B 749, 14 (2015) doi:10.1016/j.physletb.2015.07.037 [arXiv:1501.07750 [hep-ex]].

[11] G. Aad et al. [ATLAS Collaboration], Phys. Rev. D 87, no. 5, 052004 (2013) doi:10.1103/PhysRevD.87.052004 [arXiv:1211.7255 [hep-ex]].

[12] R. Aaij et al. [LHCb Collaboration], Eur. Phys. J. C 72, 2100 (2012) doi:10.1140/epjc/s10052012-2100-4 [arXiv:1204.1258 [hep-ex]].

[13] R. Aaij et al. [LHCb Collaboration], Eur. Phys. J. C 72, 2025 (2012) doi:10.1140/epjc/s10052012-2025-y [arXiv:1202.6579 [hep-ex]].

[14] S. Chatrchyan et al. [CMS Collaboration], Phys. Rev. Lett. 110, no. 8, 081802 (2013) doi:10.1103/PhysRevLett.110.081802 [arXiv:1209.2922 [hep-ex]].

[15] S. Chatrchyan et al. [CMS Collaboration], Phys. Lett. B 727, 381 (2013) doi:10.1016/j.physletb.2013.10.055 [arXiv:1307.6070 [hep-ex]].

[16] R. Aaij et al. [LHCb Collaboration], Eur. Phys. J. C 73, no. 11, 2631 (2013) doi:10.1140/epjc/s10052-013-2631-3 [arXiv:1307.6379 [hep-ex]].

[17] CMS Collaboration, CMS-PAS-BPH-15-005, http://cds.cern.ch/record/2145411

[18] CMS Collaboration, CMS-PAS-BPH-14-008, http://cds.cern.ch/record/2154307

[19] V. Khachatryan et al. [CMS Collaboration], Phys. Lett. B 753 (2016) 424 doi:10.1016/j.physletb.2015.12.020 [arXiv:1507.08126 [hep-ex]].

[20] C. Bobeth, G. Hiller and D. van Dyk, JHEP 1007, 098 (2010) doi:10.1007/JHEP07(2010)098 [arXiv:1006.5013 [hep-ph]].

[21] C. Bobeth, G. Hiller and D. van Dyk, Phys. Rev. D 87, no. 3, 034016 (2013) [Phys. Rev. D 87, 034016 (2013)] doi:10.1103/PhysRevD.87.034016 [arXiv:1212.2321 [hep-ph]].

[22] C. Bobeth, G. Hiller, D. van Dyk and C. Wacker, JHEP 1201 (2012) 107 doi:10.1007/JHEP01(2012)107 [arXiv:1111.2558 [hep-ph]].

[23] V. Khachatryan et al. [CMS Collaboration], Phys. Lett. B 757, 97 (2016) doi:10.1016/j.physletb.2016.03.046 [arXiv:1507.07527 [hep-ex]].

[24] Y. Amhis et al. [Heavy Flavor Averaging Group (HFAG) Collaboration], arXiv:1412.7515 [hepex] and online update at http://www.slac.stanford.edu/xorg/hfag 